\section{Neutralization of Osmium Tetroxide in Case of Accidental Spillage and for Disposal}

\section{K. Cooper, AIST, RT, McMaster University Medical Center}

In October 1979, a technician in the electron microscopy laboratory of one of the teaching hospitals in Toronto spilled a bottle containing approximately $500 \mathrm{ml}$ of $2 \%$ osmium tetroxide. He asked for help, and four other technicians were involved in the cleanup. They all were exposed to osmium tetroxide vapours for long enough to show signs of acute intoxication. They developed redness and swelling of the conjunctiva, impaired vision, chest pains, breathing difficulties and skin irritation and were taken into Emergency Care at the hospital. The occular symptoms disappeared after two days, but the chest pains and breathing difficulty persisted for approximately one week.

This accident could have had more serious consequences. It has been reported that exposure to osmium vapour could lead to blindness and serious respiratory trouble ${ }^{1,2}$. At McMaster University Medical Centre, the safety committee gives precise procedures to be followed in case of accidents with dangerous chemicals. All the occupants of the laboratory have to leave immediately and phone the safety and security team. The use of a self-contained breathing apparatus is advocated to re-enter the premises. Had thispolicy been followed in the Toronto hospital, the technicians would not have suffered the acute intoxication.

Recently the Government of Ontario issued an ordinance concerning disposal of dangerous chemicals. Osmium Tetroxide is amongst those, and can no longer be discarded thorough the sewage system. The ordinance, however, does not indicate how to dispose of it. For the time being, used osmium tetroxide is kept in the laboratory. This policy constitutes a hazard for the personnel. The accident in Toronto, and the new Ontario ordinance, prompted us to study ways of rapidly and effectively neutralizating osmium tetroxide

Osmium tetroxide reacts particularly with unsaturated lipid. The oxidation of the lipid double bond leads to the formation of cyclic osmic acid monoester which is hydrolized to yield diol and osmic acid. The monoester osmic acid, being unstable, reacts with the diol producing a stable diester, crosslinking two double bonds from two unsaturated aliphatic chains ${ }^{3}$.

Consequently, the feasibility of effectively neutralizing large amounts of osmium tetroxide with unsaturated lipids was studied, and the stability of the resulting diester was ascertained. Other ways of neutralizing osmium tetroxide, for example with alcohols, and with sodium hydroxide, were also investigated.

\section{Materials and Methods}

Among easily available oils, corn oil has a high percentage of unsaturated bonds ( $54 \%$ of polyunsaturated lipids in the case of Mazola Corn Oil). The determination of the amount of oil necessary to neutralize a $2 \%$ aqueous solution of osmium tetroxide was made in test tubes. Osmium tetroxide and different quantities of oil were mixed together and the presence of vapour detected with either an oil-coated glass cover slip or filter paper suspended over the solution. Blackening of the oil indicated the presence of vapor. Complete neutralization was assumed when no blackening occurred.

The following different ways of applying oil to spilled osmium tetroxide were tested:

1. Spreading ouil over the pool.

2. Applying an oil-soaked cloth.

3. Applying oil-soaked absorbent granules (cat litter)

4. Spraying oil over the pool to attempt to neutralize the vapour.

In addition, a commercial sweeping compound (Dustbane) was tested and compared to the above procedure

Finally, $2 \%$ aqueous osmium tetroxide was treated separately in test tubes with ethyl alcohol and sodium hydroxide

Osmium tetroxide that had been treated with oil was mixed with each of the following chemicals to test the stability of the diester: $1 \mathrm{~N}$ hydrochloric acid, $10 \mathrm{~N}$ hydrochloric acid, $1 \mathrm{~N}$ sulfuric acid, concentrated sulfuric acid, $50 \%$ chromic acid and hydrogen peroxide

All tests were performed in a fume hood, taking all the necessary precautions.

\section{Results}

The amount of oil needed to fully neutralize a $2 \%$ aqueous solution of osmium tetroxide, was found to be twice the volume of the osmium solution, and this ratio was used in all the tests. Complete neutralization was indicated by the oil turning black in the test tube, and by the absence of vapour as shown by the lack of oxidised black oil on the glass cover slip or filter paper above the solution.

The spreading of oil over the spilled osmium tetroxide neutralizes the fixative almost instantly, preventing the formation of vapour. This method, however, is not very convenient because of the difficulty involved in cleaning it up. Very absorbent tissue or fabric has to be used to soak up the osmium/oil mixture. Likewise, applying an oil-soaked cloth, or spraying oil over the pool, effectively neutralized the osmium tetroxide, but was messy to clean up and actually increased the spreading of the spill

The most effective method of application was found to be the oil-soaked absorbent granules. $100 \mathrm{gm}$ of granules absorbed $50 \mathrm{ml}$ of corn oil, and $300 \mathrm{gm}$ of this compound neutralizes very rapidly and effectively $50 \mathrm{ml}$ of $2 \%$ osmium tetroxide. The mixture is easy to remove, and the area easy to clean. Absorbent granules soaked in oil were kept in tightly sealed bags for more than a month, and still retained their effectiveness.

The commercial sweeping compound was ineffective at neutralizing the osmium tetroxide and is not recommended. Sodium hydroxide gave only temporary neutralization, and ethyl alcohol had no apparent effect. Neither the acids nor the hydrogen peroxide reduced the stability of the diester.

\section{Conclusion}

The present study shows that unsaturated oil is effective in neutralizing osmium tetroxide. An aqueous $2 \%$ osmium tetroxide solution is neutralized by twice its volume of oil. The diester formed by the oxidation is very stable and not destroyed by acid. Consequently, it should be easier to find a safe method of disposal for osmium tetroxide in this form. One possibility is sanitary landfill, but this is yet to be confirmed.

Used osmium tetroxide contained in a bottle can be neutralized directly with oil. Spilled osmium tetroxide should, however, be neutralized with a compound consisting of oil-soaked absorbent granules. A $2 \%$ osmium tetroxide solution will be neutralized by six times its volume of this compound.

The following recommendations are made for handling osmium tetroxide.

1. For normal use, one should never prepare more than $50 \mathrm{ml}$ of osmium tetroxide at a time.

2. The glass bottle containing the osmium tetroxide should be kept inside a padded plastic container

3. Osmium tetroxide should always be handled in a fume hood

4. All personnel in a laboratory where osmium tetroxide is used should be aware of the danger involved in handling this chemical

5. Enough oil-soaked absorbent granules should always be stored where osmium tetroxide is used

6. In case of a major spill of osmium tetroxide, the personnel should immediately leave the premises and use a self-contained breathing apparatus to reenter the laboratory, and neutralize the osmium tetroxide

7. Used osmium tetroxide should always be poured into an oil-containing bottle in order to be neutralized.

\section{Acknowledgments}

I am grateful to Dr. G. T. Simon for the helpful discussions and advice with this study. I wish to thank all members of the electron microscopy facility at McMaster University Medical Centre for their input, especially $G$. Turcon for his advice and motivation.

References

McLaughin et al 1946 Toxic manifestations of osmium tetroxide Brit. 1 . of indust Med 193

Brunot, FF :933 The toxicity of osmium terroxide (osmic acid), J. of inoust. Hyy 15 136

3. Hayat, M.A. 1970. Principles and Techniques of Electron Microscopy Biological Application.s Vol 1. Van Nostrand Reinhold Co.

Reprinted with permission. from the Microscopical Society of Canada Bulletin, 8(3) 24-28 (1988) 


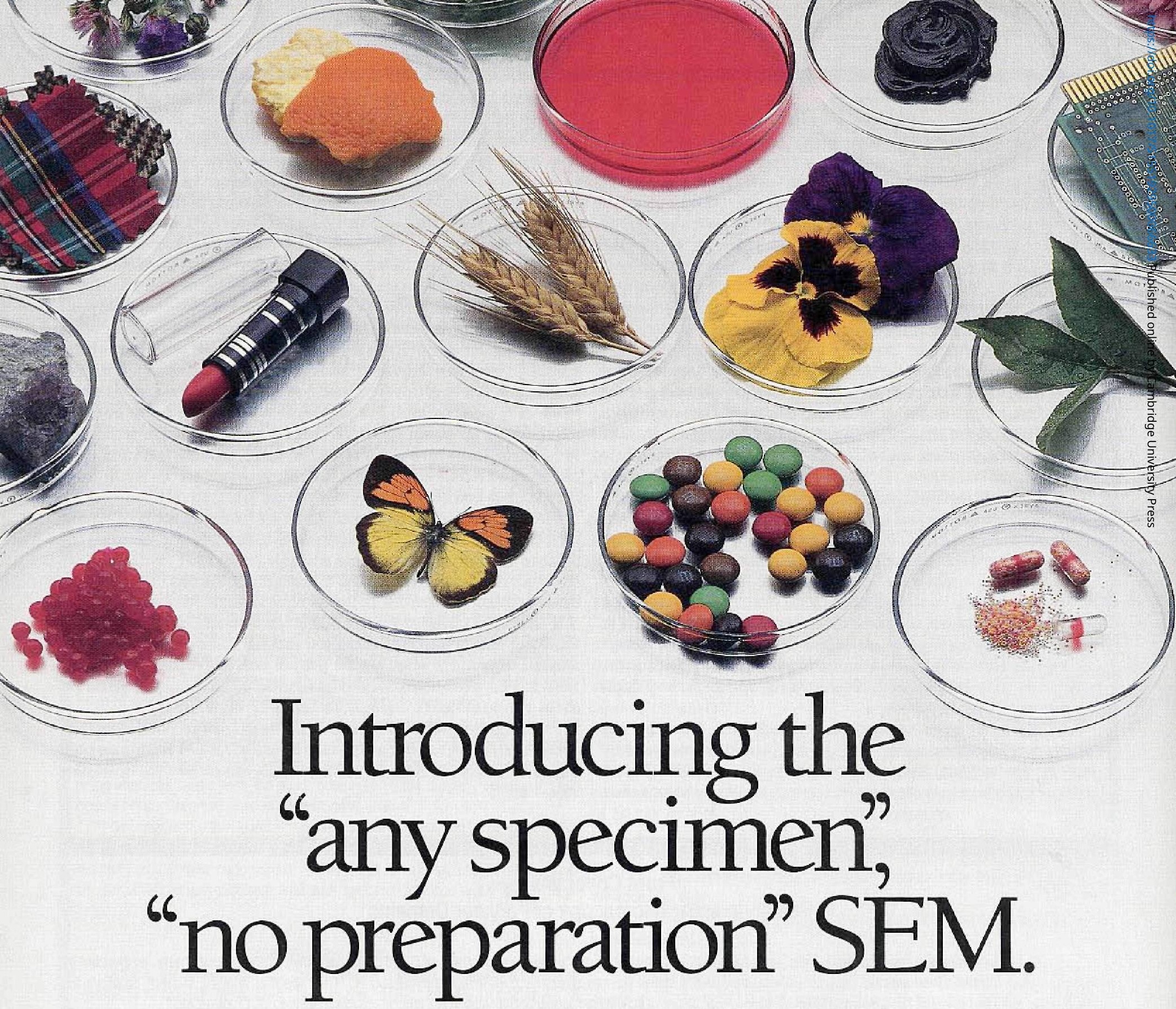

Wet specimens. Oily specimens.

Non-conductive specimens. Foods.

Pharmaceuticals. Plant life. Semiconductors. Polymers. Cosmetics. Textiles. Biological and geological specimens.

In fact, just name the specimen. We have your SEM. It's Hitachi's Variable Pressure S-2460N, the SEM that ends tedious specimen preparation such as drying or sputter coating for conductivity. Instead, specimens are observed in their natural, unaltered state - specimens up to $150 \mathrm{~mm}$ in diameter.

What's more, the S-2460N's low-vacuum mode comes without tradeoffs in performances, tradeoffs you'd face even in the costliest of other systems. At magnifications up to $200,000 \mathrm{X}$, you'll have $6.0 \mathrm{~nm}$ resolution in low-vacuum operation, $4.0 \mathrm{~nm}$ in high vacuum

Further, the $\mathrm{S}-2460 \mathrm{~N}$ is as easy to use as an optical microscope. No expertise or training needed. The vacuum system is computer controlled; high-

resolution images are obtained at the touch of a button.

More good news. This SEM costs little more than a top-notch optical microscope. And its sharp images and repeatable results come with leatures like digital frame memory and image processing; you can even add any of various analytical spectrometers.

Better call or write for details. And ask for a demonstration of the "any specimen," "no preparation" SEM. There's nothing else like it.

\section{HITACHI}

SCIENTIFIC INSTRUMENTS

Nissei Sangyo America, Ltd.

755 Ravendale Drive

Mountain View, CA 94043

(415) $969-1100$

25 West Watkins Mill Road

Gaithersburg, MD 20878

(301) $840-1650$ 
Editor's Note \#1: A "?" following your name on the address of this issue indicates that we do not know if you wish to receive this newsletter. If yow are in this category, and wish to receive the no cost newsletter, yow must complete the enclosed postage paid questionnaire to receive future issues.

Editor'\& Note \#2: In the "New And/Or Interesting in Microscopy" section of the newsietter (not included in this issue), we attempt to publish nesus of interest to microscopists - including, but not limited to, advances in technology, asuards and accomplishments, governmental and other programs, significant events, and the like we would greatly appreciate your assistance in making this a worth-while feature.

Editor's Note \#3: In this issue yow will find the first "Tricks of The Trade" articie. We invite your contributions. They may be of any length and may cover any aspect of any microscopy technique. With each accepted "T rick" as an entry, we will have a drawing at next year's MSA Conference and award a one ounce goid coin (a $\$ 400$ plus value) to the winner.

Editor's Note \#4: With the following "New Product News" as a new feature, we are attempting to provide manufacturers and suppliers with a medium in which they can announce new products, or product improvements, to the industry in a timely and inexpensive fastion.

\section{ПEW PRODUOT ПEUIS}

$\Rightarrow$ Peak Instruments has announced $\mathrm{lbe} X$, the newest addition to their spectrometer family. This application-tailored unit offers economic high performance wavelength capability for selected elements, and is full supported by Windows and Unix software. Peak has also announced the availability of the Observer, a Scanning Probe Microscope for SEMS, as a result of a strategic partnership with Topometrix. Inc. Tel.: (609)737-8133, Fax: (609)7371724. Circle Reader Inquiry \#16.

PGT has been awarded an R\&D 100 award for their PRISM ${ }^{T M}$ digital pulse processor. This innovation in $\mathrm{X}$-ray microanalysis enables the analyst to run more samples in less time with better precision and improved sensitivity to light elements.

The output of the EDS preamplifier is digitized directly, and all processing performed on the digital pulse stream. Setting can be changed continuously, and the time used to pulse shaping can even be adapted to the input signal "on the fly."

PGT is applying this technology to large area detectors to improve throughput even more - and are using it on germanium detectors to achieve the ultimate resolution resolution vs, count rate. Tel.: (609)924-7310, Fax: (609)924-1729. Circle Reader Inquiry \#17.

$\Rightarrow$ Codonics, Inc. announces the introduction of the Codonics NP-1600 Photographic Network Printer, a new 300 DPI state-of-the-art color and monochrome network printer. The NP- 1600 is a high quality paper and transparency output solution designed to work with any TCP/IP or EtherTalk based network. Utilizing dye-diffusion technology and digital image processing with 16.7 million simultaneously printable colors, the Codonics NP-1600 is capable of producing continuous tone prints ideal for multiple microscopy applications. Tel.: (800)444-1198/(216)243-1198, Fax: (216)243-1334. Circle Reader Inquiry \#18.

Polysciences, Inc. now offers high purity Tris ( $\geq 99.9 \%)$ and Tris Hydrochloride bioreagents manufactured in their GMP facility. Tris is widely used in buffers because of its buffering range and compatibility with many enzymes. The products have been tested for DNase, RNase, and protease activity and are available in small research quantities as well as in bulk. Polysciences can meet requirements for custom synthesis and packaging of these ultra pure bioreagents. Tel.: (215)343-6484, Fax: (215)343-0214. Circle Reader Inquiry \#19.

TopoMetrix announces it new on-line remote instrument operating system (RIOS) that allows scanning probe microscope (SPM) users to communicate on-line with TopoMetrix' applications and customer service staff worldwide. Tel.. (408)982-9700, Fax: (408)982-9751. Circle Reader Inquiry \#20
$\Rightarrow$ FEI Company's Components Group announces a new 2-lens electron column that offers both small spot size for high-resolution imaging (less than 20 nanometers) and high beam currents for surface analysis techniques where signal-to-noise ratios and fast acquisition times are critical. The column operates at beam voltages of $0.5 \mathrm{kV}$ to $25.0 \mathrm{kV}$ with beam currents from less than 50 picoamps to more than 200 nanoamps. It is designed for incorporation in scanning and transmission electron microscopy (SEM and TEM), Auger electron spectroscopy (AES), electron spectroscopy for chemical analysis (ESCA), micro-RHEED, and low-energy electron diffraction (LEED) systems. Tel.: (503)640-7500, Fax: (6-03)640-7509. Circle Reader Inquiry \#21.

$\Rightarrow$ The MCS system from Carl Zeiss employs a new fiber optically coupled thickness measurement technique to provide repeatable, accurate measurements of optically transparent coatings and films from 0.5 to 150 microns. White light is reflected off the front and back surfaces of the layer. The interference effects are measured and analyzed with proprietary software and frimware on an IBM PC or compatible computer. The system is fast, repeatable and easy to use, with results that are superior to current methods using micrometers, profilometers or beta backscatter techniques. Tel.: (800)2332343, Fax: (914)681-7432. Circle Reader Inquiry \#22.

Spectra-Tech, Inc. introduces a sample handling accessory that prepares microsamples for light and FT-IR microscopy: the Diamond Cleaving Knives. These versatile sample prep tools incorporate a specialized knife with a diamond cutting edge. They are useful tools for scraping and shaving off thin sections of samples. There are two diamond edge configurations. One is a straight edge, with the cutting edge on the center line of the holder. This knife is recommended for scraping a hard polymer by using a plowing motion. The other configuration is a $60^{\circ}$ angled edge with a reinforced beveled tip. It is recommended for fiber cuts and slicing. Tel.: (203)3577055. Circle Reader Inquiry \#23.

$\Rightarrow$ Leica Inc. announces the Stereoscan $420 \mathrm{C}$, a cryogenic specimen preparation system integrated with the LEICA $\$ 420$ scanning electron microscope. This innovative new system allows the preparation and transfer of frozen hydrated specimens to the SEM for viewing and analysis, including "liquid" specimens such as emulsions and suspensions. Cryopreparation also solves the problem of collapse and distortion of biological specimens which are prepared by conventional methods. Additionally, the Stereoscan $420 \mathrm{c}$ prevents loss of diffusible elements, making $x$-ray analysis more accurate. The specimens may be pre-frozen on a proprietary device or in sub-cooled liquid nitrogen using the optional Slushing Chamber and then may be transferred under protected conditions using the Transfer Device. Tel.: $(800) 248$ 0123, Fax: (708)405-0147. Circle Reader Inquiry \#24 